\title{
The Role of Intermediates in Mitochondrial Fatty Acid Oxidation
}

\author{
By KEITH K. STANLEY* and PHILIP K. TUBBS \\ Department of Biochemistry, University of Cambridge, Tennis Court Road, Cambridge CB2 1QW, U.K.
}

\author{
(Received 14 February 1975)
}

\begin{abstract}
1. Rat liver mitochondria oxidizing $\left[16-{ }^{14} \mathrm{C}\right]$ palmitoylcarnitine accumulate saturated long-chain thioester intermediates which may be detected by radio-g.l.c. 2. Time-courses of intermediate accumulation display no product-precursor relationships and the end product, measured as $\left[{ }^{14} \mathrm{C}\right]$ citrate, is produced without a detectable initial lag. 3. A short pulse of $\left[16-{ }^{14} \mathrm{C}\right]$ palmitoylcarnitine followed by unlabelled palmitoylcarnitine showed that the observed intermediates (at least in the greater part) were not the direct precursors of $\left[{ }^{14} \mathrm{C}\right]$ citrate. 4 . The quantity of saturated intermediates depended on the total accumulated flux of acyl units through the pathway provided that some mitochondrial CoA and unused substrate remained. 5 . In the presence of rotenone and carnitine, 2-unsaturated, 3-unsaturated and 3-hydroxy intermediates were formed as well as saturated intermediates. No attempt was made to detect 3-oxoacyl-CoA. 6. These observations are explained on a 'leaky-hosepipe' model of $\beta$-oxidation in which the observed intermediates arise by a constant 'leakage' from the 'true' intermediates on the pathway to citrate.
\end{abstract}

The oxidation of fatty acids in animal mitochondria occurs almost entirely by the process called $\beta$-oxidation, in which $C_{2}$ units are removed from the carboxyl end of the molecule. Each thiolytic cleavage of a $\mathbf{C}_{2}$ unit (acetyl-CoA) is preceded by the hypothetical formation of the 2-unsaturated, 3-hydroxy and 3-oxo derivatives of the acyl-CoA substrate. Some of these intermediates have been detected (as unesterified acids) during the oxidation of $\omega$-phenyl fatty acids (Dakin, 1908, 1909) and long-chain unsaturated fatty acids (Stoffel et al., 1970), but the failure to detect intermediates during the oxidation of fatty acids such as palmitate in intact systems has led to the suggestion that a multienzyme complex of fatty acid oxidation might exist (Garland \& Yates, 1967; Greville \& Tubbs, 1968). In the equally repetitive process of fatty acid synthesis the intermediates are not normally released from the enzyme (Lynen, 1961; Bressler \& Wakil, 1962).

Attempts to directly measure the intermediates of fatty acid oxidation in whole tissues (Rabinowitz \& Hercker, 1974), tissue slices (Weinhouse et al., 1944) or intact mitochondria (Garland et al., 1965) have failed. Indirect evidence has also been provided for the non-accumulation of intermediates by Weinman et al. (1950), who found no difference in the time of appearance of ${ }^{14} \mathrm{CO}_{2}$ from $\left[1-{ }^{14} \mathrm{C}\right]-,\left[6-{ }^{14} \mathrm{C}\right]-$ or $[11-$ $\left.{ }^{14} \mathrm{C}\right]$-palmitic acid (as tripalmitin) when they were injected into whole rats. Similar observations have been made for perfused heart (Evans et al., 1963) and

* Present address: Department of Medical Biochemistry, Welsh National School of Medicine, Heath Park, Cardiff CF4 4XN, U.K. for a soluble preparation from peanut cotyledons (Rebeiz \& Castelfranco, 1964). These results suggest that once breakdown is initiated, a fatty acid molecule is oxidized to completion without the release of intermediates.

In contrast with intact systems, soluble extracts and broken mitochondria are often capable of accumulating intermediates. For example these are formed by mitochondria treated with detergents (Fleming \& Hajra, 1973), sonication (Blank et al., 1971), freezethawing (Davidoff \& Korn, 1965) or incubation with fatty acid substrates in the absence of bovine serum albumin (Horak \& Pritchard, 1971). Fatty acid oxidation in soluble extracts from ox liver mitochondria has also been shown to proceed with intermediate accumulation and a consequent lag in the formation of radioactive end product from $\omega$-labelled octanoyl-CoA (Stewart et al., 1973).

Elovson (1965) showed that $10-20 \%$ of the injected $\left[3-{ }^{14} \mathrm{C}\right]$ stearate escaped as $\left[1-{ }^{14} \mathrm{C}\right]$ palmitate before oxidation to $\mathrm{CO}_{2}$ in the intact rat. In mitochondria, however, long-chain fatty acids were either totally oxidized or incorporated intact into phospholipid (Stoffel \& Schiefer, 1965). Similarly, no 3-hydroxy or medium-chain intermediates were observed in the microsomal chain-shortening of palmitoyl-CoA (Chang \& Holman, 1972). Fiecchi et al. (1973) have, however, reported a soluble chain-shortening system from rat liver which is capable of accumulating some intermediates.

A search for a multienzyme complex of fatty acid oxidation is therefore most appropriate in intact mitochondria. The pathway of fatty acid oxidation

Vol. 150 
involves the formation of four series of similar compounds, within which competitive inhibition would occur if intermediates existed in free solution. Since intermediates did not apparently accumulate in intact systems, and no differences were observed in the formation of radioactive end product from varously labelled substrates, Weinhouse et al. (1944) proposed that either (i) the activity of $\beta$-oxidation increases for short-chain substrates or (ii) complete oxidation of each substrate molecule occurs without dissociation from the enzymes. It is also possible that the restricted volume of the mitochondria could in some respects mimic the properties of a multienzyme complex, since the local steady-state concentrations of the various acyl-CoA intermediates could be rapidly reached and be sufficiently high to maintain $\beta$-oxidation. The total mitochondrial content of CoA (about $0.2 \mu \mathrm{mol} / \mathrm{g}$ wet weight of rat liver), however, is so small that no appreciable accumulation of intermediates in the whole tissue would occur unless they were exported out of the mitochondria.

Evidently, sufficiently sensitive techniques must be used to examine the state of acylation of the mitochondrial CoA. Stewart et al. (1973) have described the formation of small quantities of short-chain intermediates in mitochondria, using paper chromatography to separate the hydroxamate derivatives of radioactive acyl-CoA intermediates. Since this technique is not possible with long-chain intermediates, a radio-gas chromatograph was built to achieve the necessary sensitivity and resolution. A preliminary report of the occurrence of intermediates in mitochondrial fatty acid oxidation has already been published (Stanley \& Tubbs, 1974); the present paper describes the formation of intermediates in the presence and absence of rotenone and carnitine, and their relationship to the mechanism of $\beta$-oxidation.

\section{Materials and Methods}

\section{Materials}

$\mathrm{CoA}, \mathrm{NAD}^{+}$and ADP were obtained from Boehringer Corp. (London) Ltd., London W.5, U.K. L-Carnitine hydrochloride was obtained from KochLight Laboratories, Colnbrook, Bucks., U.K. Fatty acids and aldehydes were supplied by Fluka A.G., Buchs, Switzerland, and phenazine methosulphate was purchased from Sigma Chemical Co., St. Louis, Mo., U.S.A.

trans-Hexadec-2-enoic and tetradec-2-enoic acids were obtained from K \& K Laboratories, Plainview, N.Y., U.S.A.; silicic acid was from Mallinckrodt Chemical Works, St. Louis, Mo., U.S.A.; Hyflo Supercel was from Hopkin and Williams, Chadwell Heath, Essex, U.K. All other chemicals came from BDH Chemicals, Poole, Dorset, U.K.

$\left[1-{ }^{14} \mathrm{C}\right]$ Palmitic acid was obtained from The Radiochemical Centre, Amersham, Bucks., U.K. and $\left[16-{ }^{14} \mathrm{C}\right]$ palmitic acid was obtained from Commissariat à l'Energie Atomique, 19 Gif-surYvette, France.

Radioactive palmitoyl-L-carnitine substrates were prepared with a specific radioactivity of about $1.5 \mu \mathrm{Ci} / \mu \mathrm{mol}$ by a modification of the method of Chase \& Tubbs (1972). Inadvertent hydrolysis was eliminated by the use of thoroughly dried apparatus and by maintaining a slight excess of trifluoroacetic acid anhydride throughout the reaction as follows. The mixed anhydride of the $\left[{ }^{14} \mathrm{C}\right]$ palmitic acid and trifluoroacetic anhydride was formed in the presence of an excess of the anhydride in a $4 \mathrm{ml}$ glass-stoppered tube within a desiccator containing $\mathrm{P}_{2} \mathrm{O}_{5}$. After $2 \mathrm{~h}$ the stopper was replaced with perforated aluminium foil and the desiccator was carefully evacuated until only a white solid remained in the reaction vessel. Dry air was then introduced and a slight molar excess of $\mathrm{L}$-carnitine hydrochloride added, dissolved in trifluoroacetic acid containing $5 \%(\mathrm{v} / \mathrm{v})$ trifluoroacetic anhydride. The carnitine had been crushed in an agate mortar and dried over $\mathrm{P}_{2} \mathrm{O}_{5}$ for 7 days. After about $6 \mathrm{~h}$ at room temperature $\left(19^{\circ} \mathrm{C}\right)$ volatile reaction products were removed and the remaining viscous liquid was diluted with $1 \mathrm{ml}$ of water at $0^{\circ} \mathrm{C}$ and extracted with $3 \times 1 \mathrm{ml}$ of $n$-hexane. The aqueous fraction contained $99 \%$ of the original radioactivity. Palmitoylcarnitine was separated from unchanged palmitic acid and carnitine as described in Stanley \& Tubbs (1974).

A series of 3-hydroxy fatty acids was prepared for use as gas-chromatographic standards by the method of Angelo (1970). The melting points and i.r. spectra of the products and their methyl esters were compatible with 3-hydroxy compounds. The retention times of the methyl esters and their acetoxy derivatives after g.l.c. also agreed with reported values.

\section{Methods}

Mitochondrial experiments. Mitochondria were prepared from fresh rat liver in $0.25 \mathrm{M}$-sucrose containing $10 \mathrm{~mm}$-Tris- $\mathrm{HCl}(\mathrm{pH} 7.4)$, all operations being carried out at $0-4^{\circ} \mathrm{C}$. The liver was sliced and homogenized by hand in $8 \mathrm{vol}$. of medium. Mitochondria were then fractionated by centrifugation, taking the supernatant of a low-speed spin $(600 \mathrm{~g}, 5 \mathrm{~min})$ and the pellet after centrifugation at $6000 \mathrm{~g}$ for $15 \mathrm{~min}$. This pellet was carefully resuspended (avoiding any erythrocytes) and re-centrifuged twice. Finally sufficient medium was added to give a protein concentration of $70-90 \mathrm{mg} / \mathrm{ml}$ [measured by the method of Gornall et al. (1949)].

Oxygen-electrode incubations were carried out at $18^{\circ} \mathrm{C}$, unless otherwise stated, in a $3 \mathrm{ml}$ oxygen-electrode vessel (Rank Bros., Bottisham, Cambs., U.K.) containing $80 \mathrm{~mm}-\mathrm{KCl}, 20 \mathrm{~mm}-\mathrm{Tris}-\mathrm{HCl}$ (pH7.4), $3.3 \mathrm{~mm}-\mathrm{MgCl}_{2}, 3.3 \mathrm{~mm}$-potassium phosphate buffer (pH7.0), $1 \mathrm{~mm}$-ADP and $7 \mathrm{mg}$ of bovine serum albumin/ml [defatted by the method of Chen (1967)]. 
Table 1. Extraction procedures

Mitochondria were incubated with palmitoylcarnitine in an oxygen electrode and then treated as shown. Concentrations in parentheses are final concentrations.

'Total lipid' extraction

1. $3 \mathrm{ml} \mathrm{sample}+1 \mathrm{ml}$ wash (water) mixed with $\mathrm{KOH}(0.8 \mathrm{M})$ and kept for $2 \mathrm{~h}$ at $60^{\circ} \mathrm{C}$.

2. Acidified $(\mathrm{HCl})$ and extracted with $3 \times 5 \mathrm{ml}$ of chloroform-methanol $(2: 1, \mathrm{v} / \mathrm{v})$.

3. Dried over $\mathrm{Na}_{2} \mathrm{SO}_{4}$, evaporated and methylated.
'Hydroxamate' extraction

1. $3 \mathrm{ml} \mathrm{sample}+1 \mathrm{ml}$ wash (water) mixed with $\mathrm{HClO}_{4}(1 \%)$ and kept for $20 \mathrm{~min}$ at $0^{\circ} \mathrm{C}$.

2. Eluted from a glass-wool column $(3 \mathrm{~cm} \times 1 \mathrm{~cm})$ with $5 \mathrm{ml}$ of pyridinepropan-2-ol-water (1:1:1, by vol.) at $40^{\circ} \mathrm{C}$.

3. Eluate (containing acyl-CoA) incubated with neutral hydroxylamine $(0.5 \mathrm{M})$ for $75 \mathrm{~min}$ at $35^{\circ} \mathrm{C}$.

4. Acidified $(\mathrm{HCl})$ and extracted with $3 \times 5 \mathrm{ml}$ of diethyl ether.

5. Ether layers (hydroxamates) evaporated and incubated with $\mathrm{NaIO}_{4}(50 \mathrm{mM})$ for $20 \mathrm{~min}$ at $20^{\circ} \mathrm{C}$.

6. Acidified $(\mathrm{HCl})$ and extracted with $3 \times 5 \mathrm{ml}$ of ether.

7. Ether layers (unesterified fatty acids) dried over $\mathrm{Na}_{2} \mathrm{SO}_{4}$, evaporated and methylated.
Time-course experiments used a sampling oxygen electrode (Garland et al., 1965) with a volume of $18 \mathrm{ml}$. Incubations were terminated with $\mathrm{HClO}_{4}$ or $\mathrm{KOH}$ as shown in Table 1.

Extraction procedures. Intermediates were extracted for gas chromatography by one of two methods (Table 1). The 'total lipid' extraction procedure was designed for quantitive recovery of all (except 3-oxo) intermediates, whereas the 'hydroxamate' extraction procedure separated acyl-thioesters from mitochondrial lipids. In the latter method acyl-CoA esters were quantitatively converted into their hydroxamate derivatives at neutral $\mathrm{pH}$ in a single-phase system. This was followed by extraction and oxidative cleavage to the unesterified fatty acid by using sodium periodate (Emery \& Neilands, 1960). Both the "hydroxamate' and 'total lipid' extraction procedures gave a quantitative recovery of methyl palmitate from palmitoyl-CoA.

Chromatography of water-soluble organic acids. The distribution of radioactivity in the end products of palmitoylcarnitine oxidation was determined by ion-exchange chromatography on a column $(20 \mathrm{~cm} \times$ $1 \mathrm{~cm}$ ) of Amberlite CG400 as described by La Noue et al. (1970). This method was modified to obtain a citrate fraction from the time-course experiments (see Stewart et al., 1973), and is shown in Scheme 1.

Gas chromatography. Fatty acids were methylated and analysed by radio-g.l.c. as described by Stanley \& Tubbs (1974). Both $15 \%$ (w/v) Apiezon L and $10 \%$ $(w / w)$ ethylene glycol succinate liquid phases were used.

\section{Results}

\section{Occurrence of intermediates}

Fig. 1 shows a typical radio-gas chromatogram of the intermediates formed during $\left[16-{ }^{14} \mathrm{C}\right]$ palmitoylcarnitine oxidation in rat liver mitochondria. Sufficient bovine serum albumin was present to preserve the intact structure of the mitochondria.

The mass (upper) trace in Fig. 1 shows four major peaks, two of which correspond in retention time to the standard methyl esters $16: 0^{*}$ and $18: 0$ on both Apiezon $L$ and ethylene glycol succinate liquid phases. The 16:0 methyl ester peak is partly composed of the added substrate and corresponds to the peak of 16:0 methyl ester (shown truncated) in the radioactivity trace. Peaks (1) and (2) correspond to mixtures of unsaturated methyl esters with 18 and 20 carbon atoms respectively which are not resolved on Apiezon L. Hydrogenation converted both peaks into their corresponding saturated methyl esters.

The radioactivity (lower) trace in Fig. 1 shows the substrate (palmitate) and a series of saturated evenchain intermediates of palmitoylcarnitine oxidation. The mass corresponding to these intermediates was small compared with the mass of mitochondrial lipids in a 'total lipid' extract; consequently the mass traces have been omitted from all subsequent chromatograms. Similar results were observed in a large

* Abbreviations: $\mathrm{C}_{16: 0}$ acid, hexadecanoic acid; 16:12 acid, hexadec-2-enoic acid; 3-OH-16:0 acid, 3-hydroxyhexadecanoic acid.

Vol. 150 
$2 \mathrm{ml}$ samples mixed with

$0.5 \mathrm{ml}$ of $4 \mathrm{M}-\mathrm{KOH}$

$90 \mathrm{~min}$ at $60^{\circ} \mathrm{C}$

Cooled and $0.2 \mathrm{ml}$ of $72 \%(\mathrm{w} / \mathrm{v}) \mathrm{HClO}_{4}$ added, fatty acids extracted with $3 \times 5 \mathrm{ml}$ of chloroform-methanol

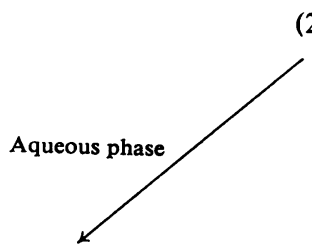

Filtered through $(3 \mathrm{~cm} \times 1 \mathrm{~cm})$ glasswool column, washed with $2 \mathrm{ml}$ of $2 \%(\mathrm{w} / \mathrm{v}) \mathrm{HClO}_{4}$, neutralized with $\mathrm{KHCO}_{3}$ and chromatographed on Amberlite CG400 micro columns

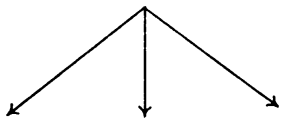

Water Formate $\mathrm{HCl}$ fraction

\section{$(2: 1, \mathrm{v} / \mathrm{v})$}

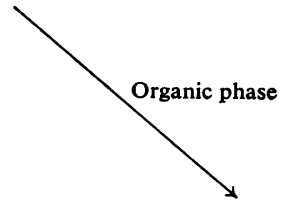

Dried with $\mathrm{Na}_{2} \mathrm{SO}_{4}$, evaporated in a stream of $\mathrm{N}_{2}$ at $60^{\circ} \mathrm{C}$ and methylated

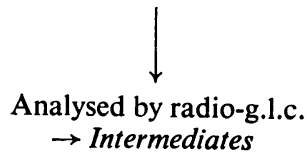

\section{Scheme 1. Time-course experiments}

During the incubation of mitochondria with $20 \mu \mathrm{M}-\left[16-{ }^{14} \mathrm{C}\right]$ palmitoylcarnitine, nine samples were treated as indicated and analysed for the substances shown in italics. For details of chromatographic procedures see the text.

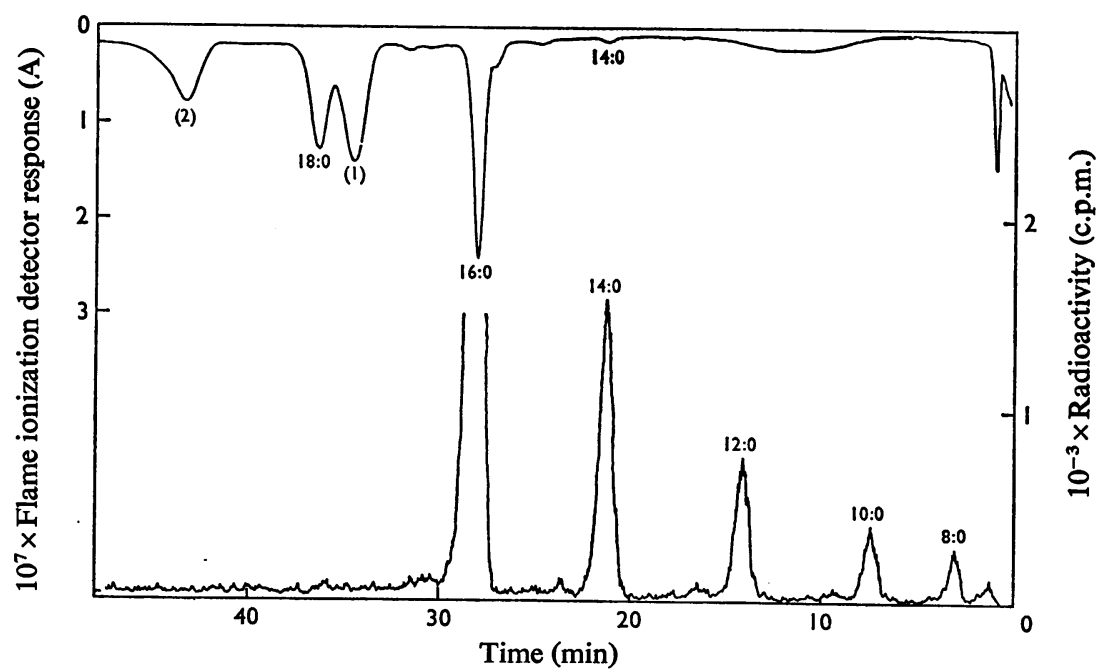

Fig. 1. Radio-gas-chromatogram of the intermediates of $\left[16-{ }^{14} \mathrm{C}\right]$ palmitoylcarnitine oxidation

Rat liver mitochondria $\left(12 \mathrm{mg}\right.$ of protein/ml) were incubated at $30^{\circ} \mathrm{C}$ in an oxygen-electrode apparatus containing the standard medium. [16- $\left.{ }^{14} \mathrm{C}\right]$ Palmitoyl-L-carnitine $(19.2 \mu \mathrm{M}, 0.09 \mu \mathrm{Ci})$ was added and the rate of $\mathrm{O}_{2}$ consumption followed until $40 \%$ of the possible $\mathrm{O}_{2}$ uptake (for conversion into acetyl-CoA) had occurred. Intermediates were extracted by the 'total lipid' extraction procedure and a sample was chromatographed on Apiezon L by using the temperature programme $5 \mathrm{~min}$ at $180^{\circ} \mathrm{C}$ followed by a linear increase in temperature of $4^{\circ} \mathrm{C} / \mathrm{min}$ to $280^{\circ} \mathrm{C}$. The upper trace is that of the flame ionization (mass) detector and below is shown the output from the proportional counter (radioactivity) ratemeter. The radioactive 16:0 methyl ester peak has been truncated and its peak height was 5200 c.p.m. Peaks (1) and (2) are described in the text. 
number of experiments using both rat liver and ox heart mitochondria.

\section{Nature of the intermediates}

Stanley \& Tubbs (1974) suggested that the observed intermediates are intramitochondrial thioesters. This hypothesis was tested by making use of the high reactivity of thioesters to hydroxylamine at neutral pH (Bruice \& Benkovic, 1966).

Fig. 2 shows the recovery of $\left[16-{ }^{14} \mathrm{C}\right]$ palmitoylcarnitine by the 'hydroxamate' extraction procedure

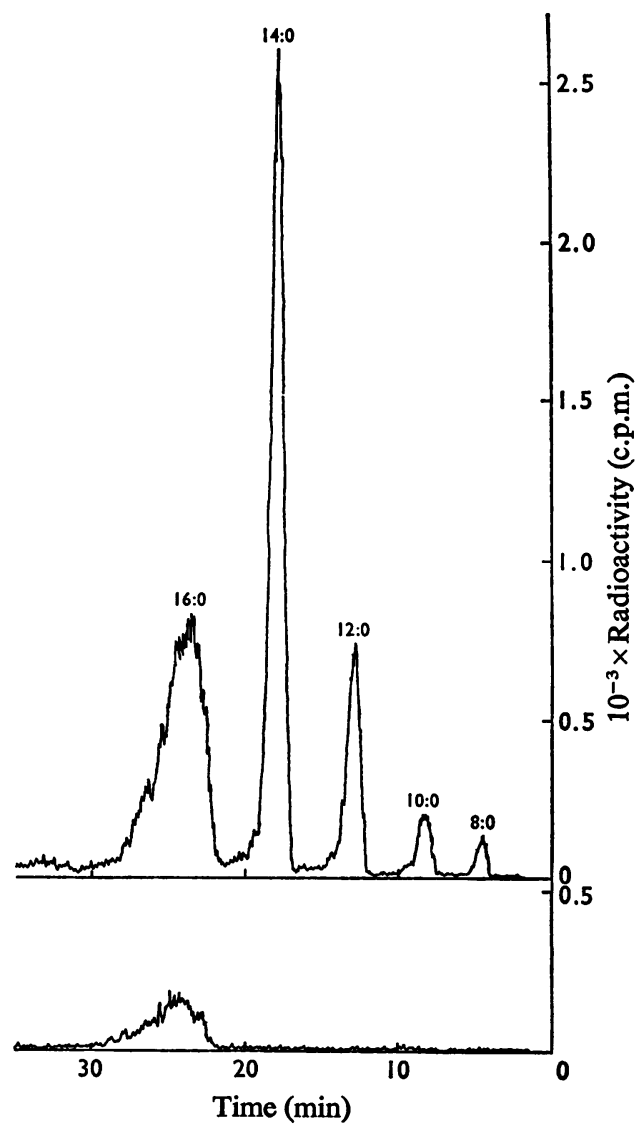

Fig. 2. Thioester nature of intermediates

Rat liver mitochondria were incubated with $19.2 \mu \mathrm{M}$ $\left[16-{ }^{14} \mathrm{C}\right]$ palmitoylcarnitine until $30 \%$ oxidation had occurred. Intermediates were then recovered by the 'hydroxamate' extraction procedure (upper trace). The lower trace shows on the same scale the recovery (less than $1 \%$ ) of an equal amount of $\left[16-{ }^{14} \mathrm{C}\right]$ palmitoylcarnitine treated in the same manner. Gas chromatography was performed on ethylene glycol succinate by a temperature programme using $5 \mathrm{~min}$ isothermal operation at $120^{\circ} \mathrm{C}$ followed by a linear rise in temperature of $4^{\circ} \mathrm{C} / \mathrm{min}$ to $190^{\circ} \mathrm{C}$. beforeandafterincubationwithrat liver mitochondria. Incubation of palmitoylcarnitine with mitochondria generated a form of palmitate which was more susceptible to aminolysis than was the substrate, as well as a series of saturated intermediates similar in pattern and size to those recovered by 'total lipid' extraction. No radioactive fatty acids were extracted into $n$-hexane at acid $\mathrm{pH}$ values before saponification or aminolysis and oxidation, suggesting that the intermediates were not unesterified acids or oxygen esters but thioesters.

\section{Time-course experiments}

Since significant quantities of saturated acyl intermediates were detected during the oxidation of palmitoylcarnitine in intact mitochondria, it seemed probable that the transport of acyl groups into the mitochondria was not rate-limiting and that a lag in the formation of radioactive end product from the $\omega$-labelled substrate should be observed. This is a diagnostic test for the accumulation of intermediates (Stewart et al., 1973), and requires the isolation of the end product of the pathway. Acetyl-CoA may accumulate to a certain extent in the mitochondrion (Garland et al., 1965). It may then condense with oxaloacetate to form citrate, or recombine to form acetoacetyl-CoA and subsequently ketone bodies. Citrate was the most convenient stable end product for analysis and so conditions were investigated for the efficient production of citrate by rat liver mitochondria. Unfortunately the terminal $\mathrm{C}_{2}$ unit of a fatty acid is preferentially incorporated into ketone bodies (Brown et al., 1954) and in this respect $\left[14^{-14} \mathrm{C}\right]$ palmitoylcarnitine would have been more suitable than the available $16-{ }^{14} \mathrm{C}$-labelled substrate. Coupled mitochondria in the presence of $1 \mathrm{~mm}$ malate produced only $48 \%$ of the possible radioactive citrate from $\left[16-{ }^{14} \mathrm{C}\right]$ palmitoylcarnitine. Uncoupling the mitochondria increased the proportion of citrate to $61 \%$, but the best citrate production was observed with coupled mitochondria in the presence of $10 \mathrm{mM}$ oxaloacetate, when citrate accounted for $74 \%$ of the radioactive products. Under these conditions the conversion of $\left[1{ }^{14} \mathrm{C}\right]$ palmitoylcarnitine into citrate was almost quantitative, showing that the activity of citrate synthase (EC 4.1.3.7) in the mitochondria was at least as great as that of $\beta$-oxidation. The rate of transport of oxaloacetate into coupled mitochondria has been reported to be rapid (Gimpel et al., 1973).

Fig. 3 shows the time-course of the end-products and of the intermediates during the oxidation of $\left[16-{ }^{14} \mathrm{C}\right]$ palmitoylcarnitine. The intermediates in Fig. 3(b) correspond to the upper line of citrate formation in Fig. 3(a), which can be seen to be linear with time for the first $90 \mathrm{~s}$. The intermediates giving rise to this linear end-product formation must be in steady state or a lag would have been observed in 

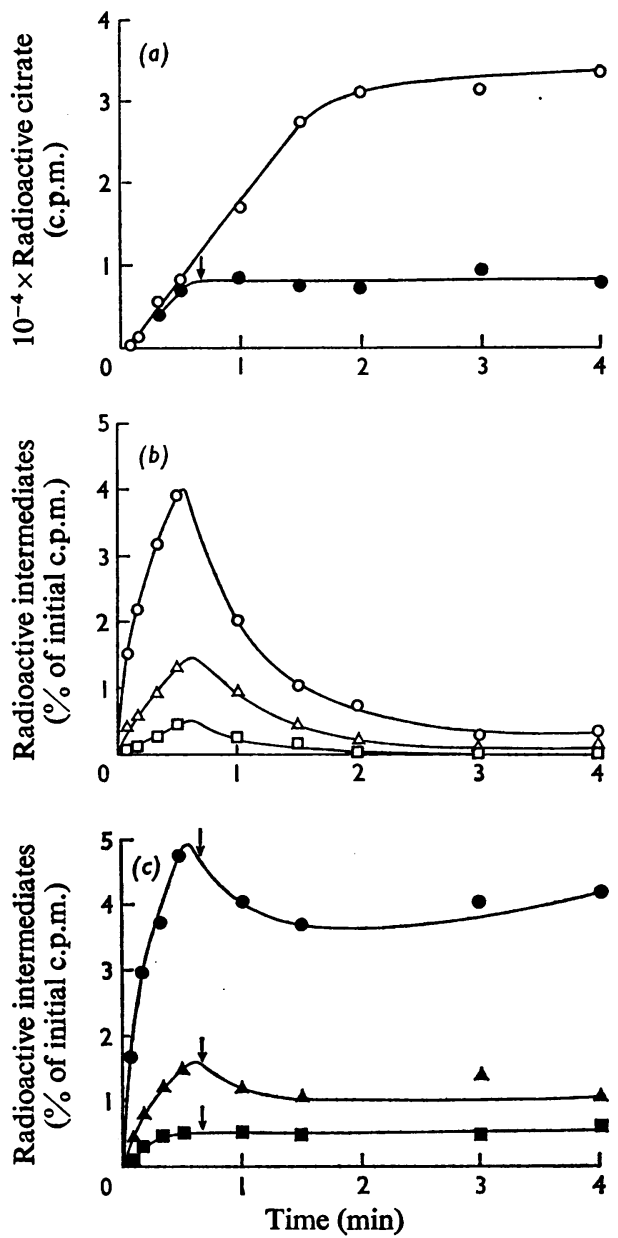

Fig. 3. Time-course of intermediates and product formation

Two separate time-course experiments were performed at $19^{\circ} \mathrm{C}$ in an $18 \mathrm{ml}$ oxygen-electrode vessel containing rat liver mitochondria $(12 \mathrm{mg}$ of protein $/ \mathrm{ml})$, standard medium, additional bovine serum albumin $(10 \mathrm{mg} / \mathrm{ml})$ and $10 \mathrm{~mm}$-oxaloacetate. In both experiments $19.2 \mu \mathrm{M}-\left[16-{ }^{14} \mathrm{C}\right]-$ palmitoylcarnitine was added at zero time. In the second experiment $(\Theta, \Lambda, \Xi) 200 \mu \mathrm{M}$ unlabelled palmitoylcarnitine was added after $40 \mathrm{~s}$, as indicated by the arrows. Watersoluble organic acids were separated as shown in Scheme 1 and the radioactivity in the citrate fraction is shown in $(a)$; $O$, normal incubation; $O$, with a chase of unlabelled substrate. The time-courses of radioactive intermediates (measured by radio-g.l.c.) corresponding to these two incubations are shown in $(b)$ and $(c)$ respectively; $O, 0,14: 0$; $\Delta, \Delta, 12: 0, \square, \square, 10: 0$. Some citrate was lost during the chloroform-methanol $(2: 1, v / v)$ extraction as shown in Scheme 1 and the results in $(a)$ cannot therefore be expressed as a percentage of the radioactivity added in the substrate ( $\%$ of initial c.p.m.). In a parallel experiment using $n$-hexane to extract intermediates, radioactive citrate was formed at about four times the rate of the radioactive 14:0 methyl ester $(120 \mathrm{nmol} / \mathrm{min})$, but, under these conditions, the recovery of intermediates was impaired. the accumulation of citrate. Fig. 3(b) shows that intermediates did accumulate but that they were not at steady-state values. Further, the intermediates did not bear a product-precursor relationship to one another as would be expected if they were 'free' intermediates on the pathway of citrate formation. Instead each intermediate reached a maximum value at about the same time. This suggests that the intermediates' observed by radio-g.l.c. are not the direct precursors of $\left[{ }^{14} \mathrm{C}\right]$ citrate but arise by a slow reversible process from smaller pools of 'true' intermediates. That is, while substrate oxidation is rapid 'intermediate' pools accumulate, but when the substrate is exhausted these 'intermediates' may feed back into $\beta$-oxidation. The initial rate of $\left[{ }^{14} \mathrm{C}\right]$ citrate formation was about four times that of the radioactive intermediates.

The presence of two pools of intermediates was confirmed by the addition of a 'chase' of unlabelled substrate during the oxidation of $\left[16-{ }^{14} \mathrm{C}\right]$ palmitoylcarnitine. The lower line in Fig. 3(a) demonstrates the immediate dilution of radioactive isotope in the end product (and presumably in the 'true' intermediates also) after the addition of the chase of unlabelled substrate. The 'intermediates' observed by radio-g.l.c., however, do not show a rapid dilution of radioactivity but are in fact maintained at high values (Fig. 3c). Since the CoA content of the mitochondria is limited it is unlikely that the immediate attenuation of radioactive citrate formation could have been due to the formation of large pools of unlabelled acyl-CoA.

\section{Quantitative significance of the intermediates}

The concentration of $\mathrm{C}_{10}-\mathrm{C}_{14}$ intermediates in Fig. 1 was about $0.2 \mathrm{nmol} / \mathrm{mg}$ of mitochondrial protein. This value, which should be increased to allow for palmitoyl-CoA, is comparable with the quantity of $\mathrm{HClO}_{4}$-insoluble (long-chain) acyl-CoA measured under similar conditions by Garland et al. (1965) and by Chase \& Tubbs (1972), and shows that most of the long-chain acyl-CoA has become labelled.

A series of incubations was carried out with decreasing amounts of mitochondria (Table 2). Palmitoylcarnitine was added and the oxidation allowed to proceed (at a rate approximately inversely related to the protein concentration) until about $40 \%$ of the theoretical $\mathrm{O}_{2}$ consumption had occurred. Radiog.l.c. revealed that approximately equal amounts of the radioactive 14:0 methyl ester had been formed at the two highest mitochondrial concentrations (Table 2), despite a 10-fold difference in the amount of mitochondria. At first sight this might imply that the observed intermediates were not associated with the mitochondria. If they arise by slow dissociation from smaller pools of 'true' intermediates, however, then their accumulation would be expected to depend on the total amount of substrate that had been oxi- 
Table 2. Intermediate formation by decreasing amounts of mitochondria

Various amounts of rat liver mitochondria were incubated in a $3 \mathrm{ml}$ oxygen-electrode apparatus at $18^{\circ} \mathrm{C}$. Equal amounts of bovine serum albumiti and $\left[16-{ }^{14} \mathrm{C}\right]$ palmitoylcarnitine were added in each case and the oxidation was followed for various times until about $40 \%$ of the possible $\mathrm{O}_{2}$ consumption had occurred. Samples were then deproteinized in $\mathrm{HClO}_{4}$ and un-

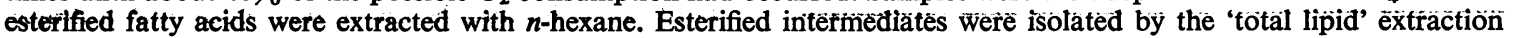
procedure and the radioactive $14: 0$ methyl ester estimated by radio-g.l.c.

$\begin{array}{cccccc}\text { Sample } & \begin{array}{c}\text { Protein } \\ (\mathrm{mg})\end{array} & \begin{array}{c}\text { Oxidation } \\ (\%)\end{array} & \begin{array}{c}\text { Time } \\ (\text { min) }\end{array} & \overbrace{(\mathrm{nmol})}^{\text {Radioactive 14:0 }} & \begin{array}{c}\text { (nmol/mg) } \\ 1\end{array} \\ 37.5 & 39 & 1.0 & 2.28 & 0.061 \\ 2 & 3.75 & 41 & 8.5 & 2.64 & 0.70 \\ 3 & 1.88 & 39 & 15.0 & 1.89 & 1.00 \\ 4 & 0.75 & 38 & 64.0 & 1.08 & 1.45\end{array}$
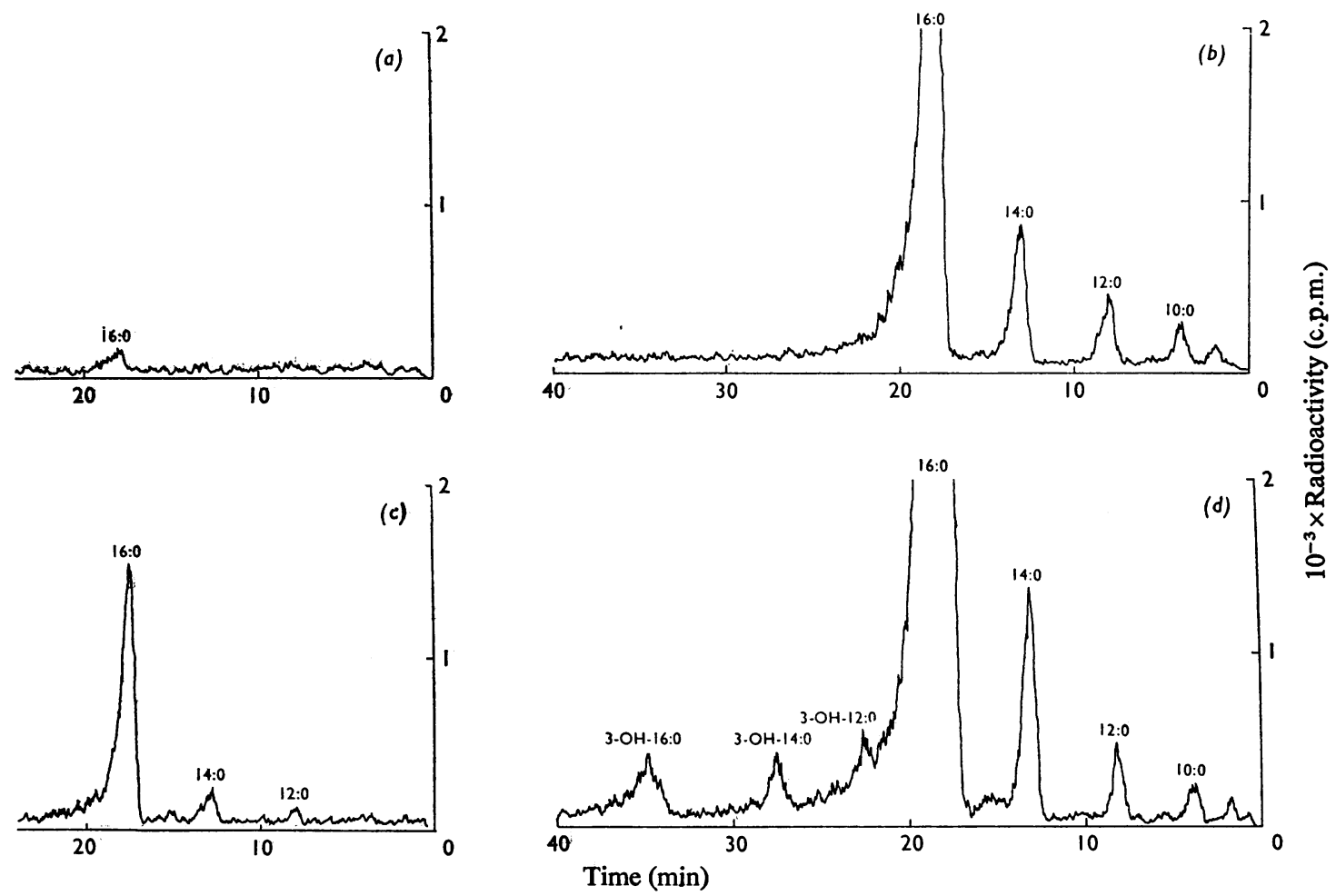

Fig. 4. Effect of dilution on intermediate formation

The unesterified fatty acids (after methylation) and intermediates isolated as described in the legend to Table 2 were chromatographed on ethylene glycol succinate by a temperature programme using $5 \mathrm{~min}$ isothermal operation at $120^{\circ} \mathrm{C}$ followed by a linear rise in temperature of $4^{\circ} \mathrm{C} / \mathrm{min}$ to $190^{\circ} \mathrm{C}$. (a) and $(b)$, Sample 1 in Table $2 ;(c)$ and $(d)$, sample 4. (a) and $(c)$, the unesterified fatty acids; $(b)$ and $(d)$, the esterified intermediates. Only one-third of sample 1 was injected on to the g.l.c.

dized. Intermediate formation would only cease when nearly all the intramitochondrial $\mathrm{CoA}$ becomes acylated. This effect is seen at the two lower mitochondrial concentrations in Table 2 when the combined interniediate value approaches the mitochond- rial $\mathrm{CoA}$ content of some $2 \mathrm{nmol} / \mathrm{mg}$ of protein. Fig. 4 shows the appearance of the intermediates at two protein concentrations with their corresponding hexane extracts. Evidently after long periods of incubation some unesterified fatty acids were generated, 
but even then they represent only a small fraction of the total radioactive intermediates. 3-Hydroxy intermediates also accumulated in small amounts after long incubation times.
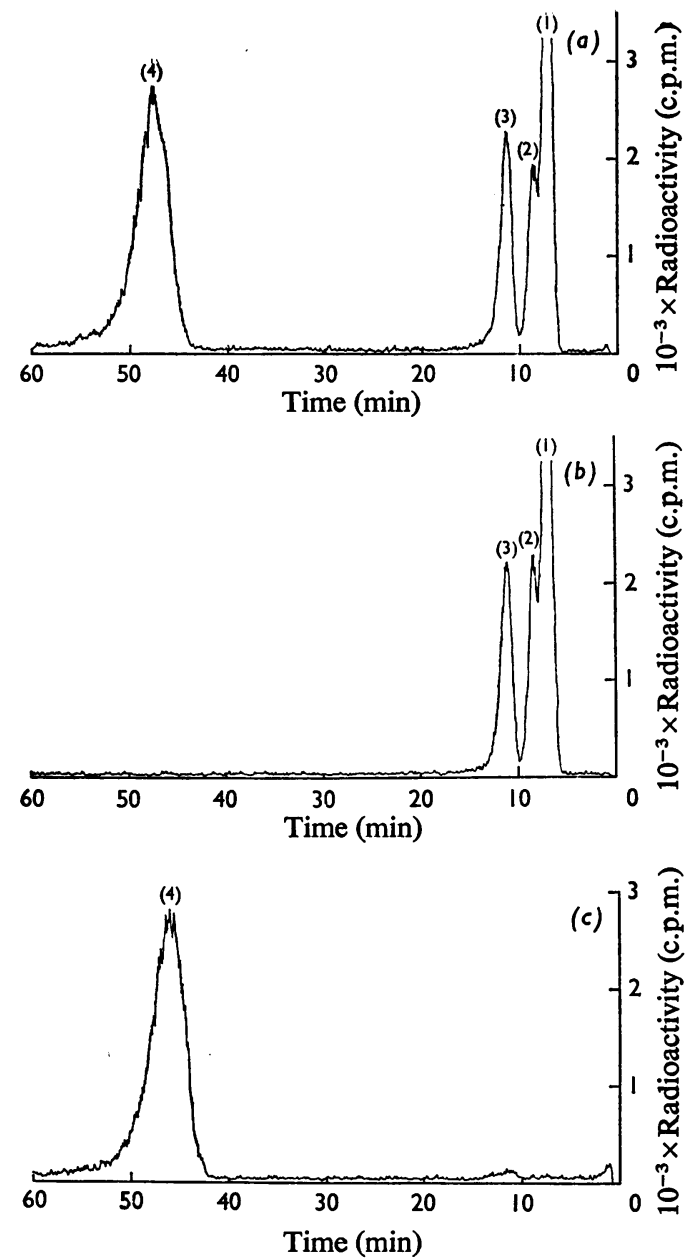

Fig. 5. Intermediates formed in the presence of rotenone and carnitine

Rat liver mitochondria ( $11 \mathrm{mg}$ of protein/ml) were preincubated in standard oxygen-electrode medium with $10 \mathrm{~mm}$-carnitine and $42 \mu \mathrm{M}$-rotenone for $3 \mathrm{~min}$ at $18^{\circ} \mathrm{C}$. $\left[1-{ }^{14} \mathrm{C}\right]$ Palmitoylcarnitine $(24 \mu \mathrm{M})$ was added and after $8 \mathrm{~min}$ incubation the intermediates were isolated by the 'total lipid' extraction procedure. (a) Fraction of 'total lipid' extract. Most of the methyl esters were applied to a column $(12 \mathrm{~cm} \times 0.5 \mathrm{~cm})$ of silicic acid containing $33 \%$ (by wt.) of Hyflo Supercel. (b) Methyl esters eluted with $6 \mathrm{ml}$ of $n$-hexane containing $2 \%(\mathrm{v} / \mathrm{v})$ of diethyl ether. Polar methyl esters $(c)$ were eluted with $6 \mathrm{ml}$ of diethyl ether. Each sample was analysed by radio-g.l.c. on ethylene glycol succinate at $170^{\circ} \mathrm{C}$. The radioactive peaks were identified by acetylation, hydrogenation and oxidative cleavage as the following methyl esters: (1) 16:0, (2) $16: 1^{3}$, (3) $16: 1^{2}$, (4) $3-\mathrm{OH}-16: 0$.

\section{Experiments with rotenone and carnitine}

The accumulation of significant quantities of longchain saturated acyl intermediates under normal conditions highlights the almost complete absence of the 3-hydroxy and 2-unsaturated intermediates. The recovery of 3-oxoacyl-CoA was not expected to be high after hydrolytic extraction, and no attempt was made to recover the decarboxylated product, which for 3-oxohexadecanoic acid would be 2-oxopentadecane. Partial inhibition of mitochondrial fatty acid oxidation by pent-4-enoate (Senior \& Sherratt, 1968) and 2-bromopalmitoylcarnitine (Chase \& Tubbs, 1972) did not cause the formation of unsaturated or hydroxy intermediates (results not shown). A different pattern was produced by rotenone, however, which specifically inhibits NADH oxidation and hence the 3-hydroxyacyl-CoA dehydrogenase reaction. Bremer \& Wojtczak (1972) have already reported the accumulation of 3-hydroxyacylcarnitine by intact mitochondria in the presence of rotenone and carnitine.

Fig. 5 shows the radioactive products that accumulated during the oxidation of $\left[1-{ }^{14} \mathrm{C}\right]$ palmitoylcarnitine under these conditions; the $1-{ }^{14} \mathrm{C}$-labelled substrate was used so that only $\mathbf{C}_{16}$ intermediates would be labelled. Figs. 5(b) and 5(c) show the separation of these intermediates on a column of silicic acid into three non-polar methyl esters [eluted in hexane containing $2 \%(\mathrm{v} / \mathrm{v})$ of diethyl ether] and one polar methyl ester (eluted in diethyl ether). The retention time of the polar methyl ester (peak 4) corresponded to synthetic methyl 3-hydroxypalmitate and was in close agreement with the relative retention time (6.60) with respect to the corresponding saturated compound reported by Davidoff \& Korn (1964) and Barron \& Mooney (1970). After acetylation, a good correspondence with the retention time of synthetic methyl 3-acetoxypalmitate was also obtained.

The non-polar methyl esters contained the substrate (peak 1) and two peaks that were hydrogenated by treatment with $\mathbf{H}_{2}$ and $\mathbf{P d C l}_{2}$. Peak (3) was also hydrogenated by zinc dust and $\mathrm{HCl}$ [which reduces only 2-unsaturated methyl esters (Rosenfelder et al., 1974)] and had a relative retention time of 1.63 , which identifies it as the trans-2-unsaturated methyl ester. The relative retention time of peak (2) (1.24) was close to that reported by Barron \& Mooney (1970) for the 3-unsaturated ester (1.27) and was considerably different from either the cis-2-unsaturated (1.04) or the 9-unsaturated (1.18) methyl esters. Oxidative cleavage by the method of Von Rudolf (1956) of a fraction collected by gas chromatography from the products of $\left[16-{ }^{14} \mathrm{C}\right]$ palmitoylcarnitine oxidation (shown in Fig. 6) produced a 13:0 acid fragment, confirming the presence of methyl hexadec-3-enoate. Fig. 6 shows the pattern of intermediates during 


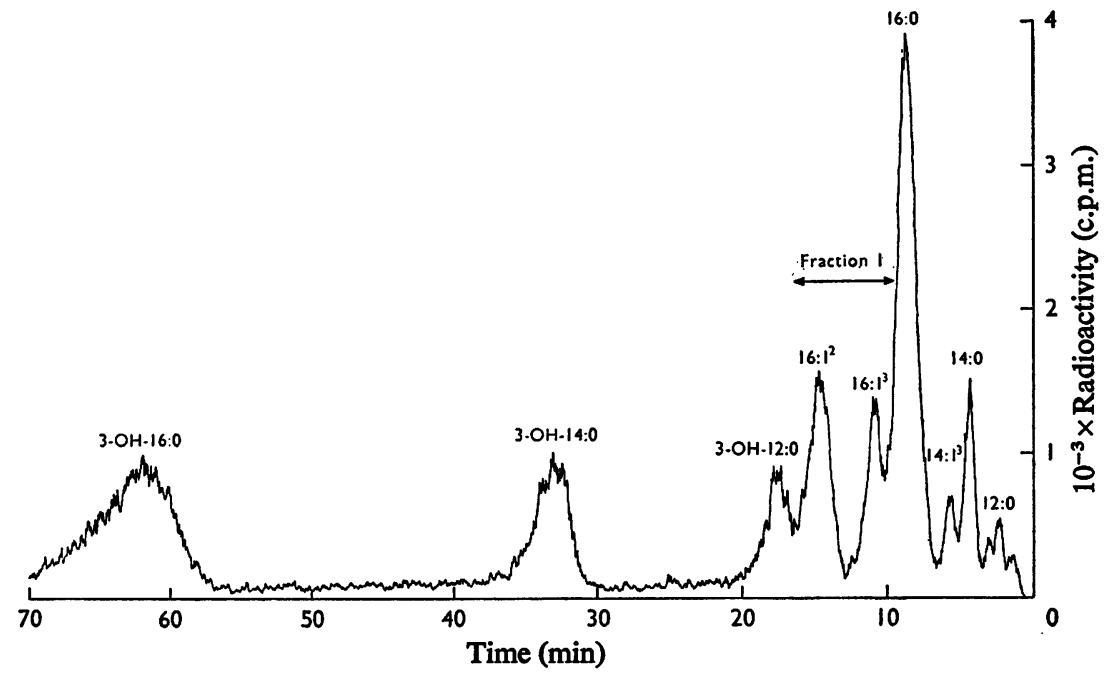

Fig. 6. Effect of rotenone and carnitine on the oxidation of $\left[16-{ }^{14} \mathrm{C}\right]$ palmitoylcarnitine

Rat liver mitochondria $(14 \mathrm{mg}$ of protein $/ \mathrm{ml})$ were allowed to oxidize $19.2 \mu \mathrm{M}-\left[16-{ }^{14} \mathrm{C}\right]$ palmitoylcarnitine after $3 \mathrm{~min}$ preincubation with $42 \mu \mathrm{M}$-rotenone and $10 \mathrm{~mm}$-carnitine. Bovine serum albumin $(7 \mathrm{mg} / \mathrm{ml})$ was added just before the substrate. Intermediates were isolated by the 'total lipid' extraction procedure and gas chromatography was performed on ethylene glycol succinate at $160^{\circ} \mathrm{C}$. Fraction 1 shows the position of a fraction collected for analysis of double-bond position by the method of Von Rudolf (1956).

[16- $\left.{ }^{14} \mathrm{C}\right]$ palmitoylcarnitine oxidation in the presence of rotenone and carnitine as identified above.

Methyl hexadec-3-enoate was not produced by isomerization of methyl hexadec-2-enoate or 3hydroxypalmitate on either ethylene glycol succinate or silicic acid columns. Hydrolysis of the $16: 1^{2}$ methyl esters or acyl-CoA in methanolic $\mathrm{NaOH}$ has been reported to cause some formation of the 3methoxy acid but not the 16:13 acid (Barron \& Mooney, 1970). No methanol was used in these experiments and synthetic $16: 1^{2}$ acid was unaffected by the hydrolytic conditions of the 'total lipid' extraction procedure. It appears therefore that $16: 1^{3}$ (presumably as its CoA ester) is a product of oxidation of palmitoylcarnitine in rat liver mitochondria in the presence of rotenone.

\section{Discussion}

The failure to detect intermediates during the oxidation of ordinary fatty acids in intact tissues has led to the suggestion that a multienzyme complex may exist which functions without the formation of free intermediates. The present results, however, extend the preliminary report by Stanley \& Tubbs (1974) of saturated intramitochondrial acyl intermediates during palmitoylcarnitine oxidation. These intermediates have been shown to be thioester in nature. Unesterified fatty acids were only generated in small quantities after prolonged incubation. Synthesis of intermediates from $\left[{ }^{14} \mathrm{C}\right]$ acetyl-CoA de novo was not observed in experiments using $\left[1-{ }^{14} \mathrm{C}\right]$ palmitoylcarnitine and no chain-elongation of palmitate occurred. The lack of product-precursor relationships between adjacent intermediates strongly suggests that they have a mitochondrial localization rather than being formed in free solution by a contaminating population of broken mitochondria, which would, in any case, be incapable of significant oxidation of palmitoylcarnitine in the absence of added CoA and $\mathrm{NAD}^{+}$. The time-course experiments suggested that the observed intermediates were not the direct precursors of citrate produced by fatty acid oxidation but that they arose from separate smaller pools of 'true' intermediates by a process that was slow with respect to the rate of citrate formation, and also reversible.

An interpretation of these results is shown in Scheme 2. The oxidation of palmitoylcarnitine involves 27 hypothetical intermediates in seven repetitive cycles, of which only the first three are shown. At the end of each cycle an acetyl-CoA molecule is released, but only after seven cycles is labelled acetylCoA formed from $\left[16^{14} \mathrm{C}\right]$ palmitoylcarnitine. This $\left[{ }^{14} \mathrm{C}\right] a c e t y l-C o A$ (measured as citrate) was formed linearly with time; therefore, since there is no evidence of a rate-limiting step at the end of $\beta$-oxidation or at citrate synthase, the intermediates giving rise to citrate must have reached steady-state concentrations 
'Free' intermediates

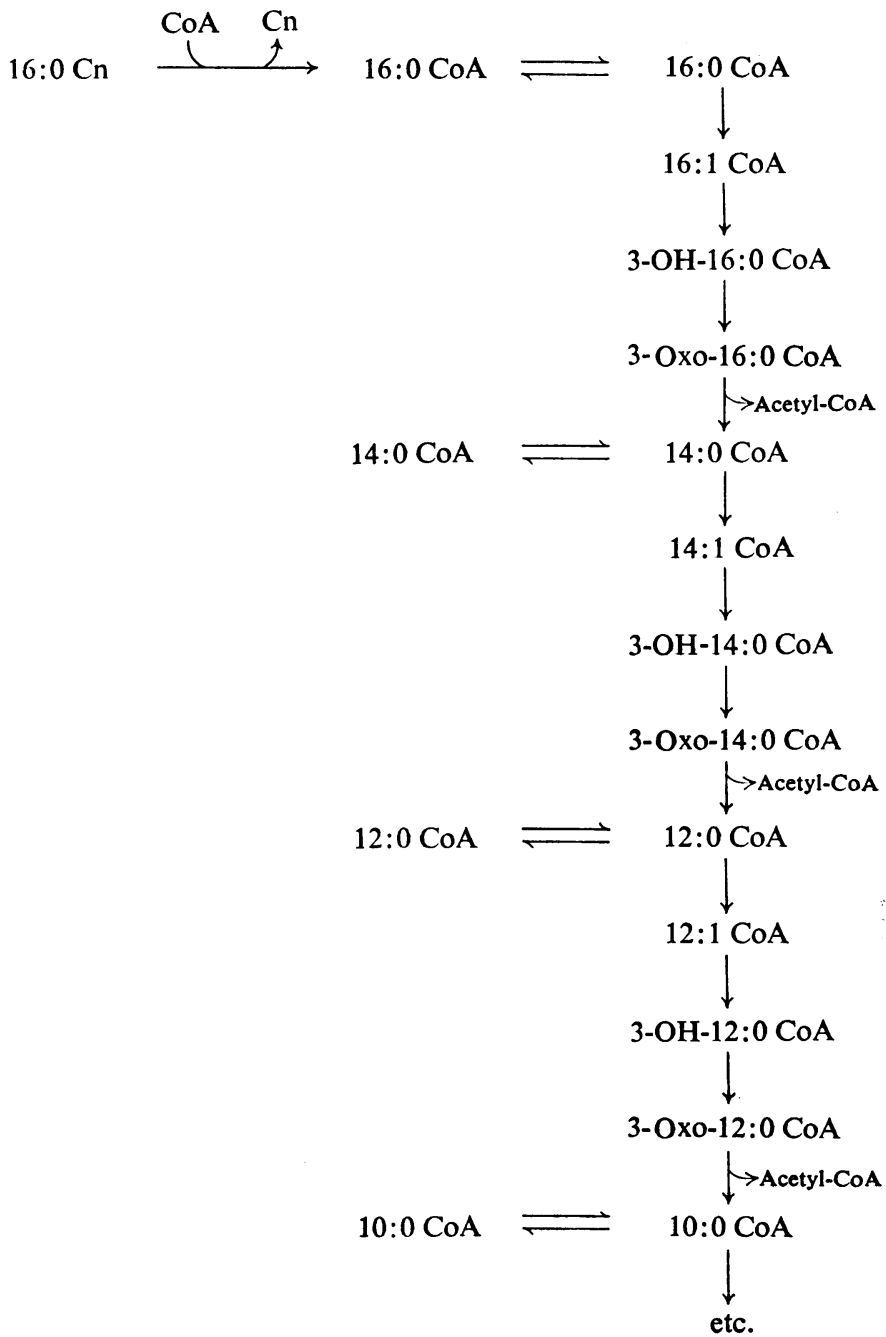

Scheme 2. A model of $\beta$-oxidation

Palmitoylcarnitine $(16: 0 \mathrm{Cn})$ is converted into palmitoyl-CoA $(16: 0 \mathrm{CoA})$ at the inner mitochondrial membrane. Two pools of acyl-CoA intermediates are shown labelled as 'free' and 'true' intermediates. The 'true' intermediates are the postulated direct precursors of citrate produced by $\beta$-oxidation. They do not appear to be in rapid equilibrium with the surrounding medium but give rise to a second larger pool of 'free' intermediates in the mitochondria. The latter intermediates are observed by radio-g.l.c. to accumulate relatively slowly, and the terminal acetyl-CoA is produced linearly with time.

before the first time-point on the measured timecourse. The intermediates detected by radio-g.l.c., however, accumulated relatively slowly during rapid substrate oxidation and then decreased as the substrate became exhausted. The quantity of intermediates observed apparently depended on the total flux of acyl units through $\beta$-oxidation that had occurred, rather than on the amount of mitochondrial protein in the incubation, provided that some free mitochondrial CoA and unused substrate remained. In Scheme 2, separate pools of 'free' intermediates are postulated to account for these results. Since the observed intermediates are supposed to arise by leakage from the 'true' intermediates (which are restricted in amount and have privileged access to the enzymes of $\beta$-oxidation) they do not create a lag 
in the end-product formation or show productprecursor relationships. This model is similar to a 'leaky hosepipe' in which water flows through a horizontal hosepipe with constricted vertical side arms. While water emerges at a constant rate from the hosepipe (analogous to citrate formation) the levels in the side arms (the observed intermediates) simultaneously rise until they eventually reflect the gradation of hydrostatic pressure along the hosepipe. The presence of two pools of intermediates was confirmed by the pulse-chase radioactivity experiments.

Under normal conditions, no 3-hydroxy or 2unsaturated compounds were detected, even though these must have been the precursors of the observed saturated intermediates. Only in the presence of rotenone (or after prolonged incubation) were these intermediates observed. Carnitine was added to incubations containing rotenone to facilitate the export of 'free' acyl intermediates as carnitine esters and to increase the size of the observed peaks, although no new peaks were formed by carnitine either in the presence or in the absence of rotenone. Rotenone also caused the appearance of the 3-unsaturated acyl derivatives. Hexadec-3-enoyl-CoA has been observed as an intermediate of fatty acid metabolism in preparations of cellular slime mould (Dictyostelium discoideum) and damaged guinea-pig mitochondria (Davidoff \& Korn, 1964, 1965), and a mitochondrial enzyme catalysing the isomerization of 2- and 3-unsaturated acyl-CoA has been described by Stoffel et al. (1970).

One problem with Scheme 2 is that the oxidation of 'free' palmitoyl-CoA must be as rapid as the flux through $\beta$-oxidation to citrate, while the oxidation of other acyl-CoA pools must be considerably slower than their formation while they are still growing in size. It is possible that external acyl-carnitine has a privileged access to the pathway of $\beta$-oxidation compared with internal acyl-CoA, thus effectively bypassing the intermediate pool of 'free' palmitoylCoA shown in Scheme 2.

The pathway of $\beta$-oxidation, like those of fatty acid synthesis and protein synthesis, has peculiar problems of organization owing to the repetitive nature of the reactions involved. Each of the 27 'true' intermediate pools has to be traversed by the rapid flux of $\beta$-oxidation, yet there is no evidence that these intermediates accumulate despite their competitive nature. The physical interpretation of the 'leaky-hosepipe' model is not clear, but evidently $\beta$-oxidation in mitochondria does not occur by either a mechanism in which all intermediates accumulate in the total matrix space, or alternatively by a multienzyme complex from which no intermediates whatsoever are released. The concept of a multienzyme complex must be modified to allow for the 'leakage' of saturated intermediates, but complete release of these from the $\beta$-oxidation system cannot occur since they show no product-precursor relationships. A decreasing $\boldsymbol{K}_{\boldsymbol{m}}$ value for shorter-chain intermediates could decrease the amounts of intermediates observed, but would not remove their product-precursor relationships. The observation of two pools of intermediates on the other hand suggests that a mechanism of 'free' intermediates must be located within a micro compartment. It may be noted that this model resembles the theoretical case, considered by Goldman \& Katchalski (1971), of two enzymes catalysing consecutive reactions and situated in an unstirred microenvironment. The release of final product into the bulk (stirred) solution is able to proceed without a lag in such cases, and the leak of intermediates is relatively slow. The evidence for the 'leaky-hosepipe' model cannot distinguish between these two interpretations.

K. K. S. and P. K. T. thank the Medical Research Council and the Science Research Council respectively for financial support.

\section{References}

Angelo, B. (1970) Bull. Soc. Chim. Fr. 5, 1848-1849

Barron, E. J. \& Mooney, L. A. (1970) Biochemistry 9, 2143-2152

Blank, M. L., Cress, E. A., Stephens, N. \& Snyder, F. (1971) J. Lipid Res. 12, 638-640

Bremer, J. \& Wojtczak, A. B. (1972) Biochim. Biophys. Acta 280, 515-530

Bressler, R. \& Wakil, S. J. (1962) J. Biol. Chem. 237, 14411448

Brown, G. W., Chapman, D. D., Matheson, H. R., Chaikoff, I. L. \& Dauben, W. G. (1954) J. Biol. Chem. 209, 537-548

Bruice, T. C. \& Benkovic, S. J. (1966) Bioorganic Mechanisms, vol. 1, chapter 3, Benjamin, New York

Chang, H. \& Holman, R. T. (1972) Biochim. Biophys. Acta 280, 17-21

Chase, J. F. A. \& Tubbs, P. K. (1972) Biochem. J. 129, 55-65

Chen, R. F. (1967) J. Biol. Chem. 242, 173-181

Dakin, H. D. (1908) J. Biol. Chem. 4, 419-435

Dakin, H. D. (1909) J. Biol. Chem. 6, 221-223

Davidoff, F. \& Korn, E. D. (1964) J. Biol. Chem. 239, 2496-2506

Davidoff, F. \& Korn, E. D. (1965) J. Biol. Chem. 240, 1549-1558

Elovson, J. (1965) Biochim. Biophys. Acta 98, 36-40

Emery, T. \& Neilands, J. B. (1960) J. Am. Chem. Soc. 82, 3658-3662

Evans, J. R., Opie, L. H. \& Shipp, J. C. (1963) Am. J. Physiol. 205, 766-770

Fiecchi, A., Galli-Kienle, M., Scala, A., Galli, G. \& Paoletti, R. (1973) Eur. J. Biochem. 38, 516-528

Fleming, P. J. \& Hajra, A. K. (1973) Biochem. Biophys. Res. Commun. 55, 743-751

Garland, P. B. \& Yates, D. W. (1967) in Mitochondrial Structure and Compartmentation (Quagliariello, E., Papa, S., Slater, E. C. \& Tager, J. M., eds.), pp. 385-389, Adriatica Editrice, Bari 
Garland, P. B., Shepherd, D. \& Yates, D. W. (1965) Biochem. J. 97, 587-594

Gimpel, J. A., De Haan, E. J. \& Tager, J. M. (1973) Biochim. Biophys. Acta 292, 582-591

Goldman, R. \& Katchalski, E. (1971) J. Theor. Biol. 32, 243-257

Gornall, A. G., Bardawill, C. J. \& David, M. M. (1949) J. Biol. Chem. 177, 751-766

Greville, G. D. \& Tubbs, P. K. (1968) Essays Biochem. 4, 155-212

Horak, H. \& Pritchard, E. T. (1971) Biochim. Biophys. Acta 248, 515-519

La Noue, K., Nicklas, W. J. \& Williamson, J. R. (1970) J. Biol. Chem. 245, 102-111

Lynen, F. (1961) Fed. Proc. Fed. Am. Soc. Exp. Biol. 20, 941-951

Rabinowitz, J. L. \& Hercker, E. S. (1974) Arch. Biochem. Biophys. 161, 621-627
Rebeiz, C. \& Castelfranco, P. (1964) Plant Physiol. 39, 932-938

Rosenfelder, G., Lüderitz, O. \& Westphal, O. (1974) Eur. J. Biochem. 44, 411-420

Senior, A. E. \& Sherratt, H. S. A. (1968) Biochem. J. 110, 521-527

Stanley, K. K. \& Tubbs, P. K. (1974) FEBS Lett. 39, 325328

Stewart, H. B., Tubbs, P. K. \& Stanley, K. K. (1973) Biochem. J. 132, 61-76

Stoffel, W. \& Schiefer, H. (1965) Hoppe-Seyler's Z. Physiol. Chem. 341, 84-90

Stoffel, W., Ecker, W., Assad, H. \& Sprecker, H. (1970) Hoppe-Seyler's Z. Physiol. Chem. 351, 1545-1554

Von Rudolf, E. (1956) Can. J. Chem. 34, 1413-1418

Weinhouse, S., Medes, G. \& Floyd, N. F. (1944) J. Biol. Chem. 155, 143-151

Weinman, E. O., Chaikoff, I. L., Dauben, W. G., Gee, M. \& Entenman, C. (1950) J. Biol. Chem. 184, 735-744 